\title{
DIE KOMPETENZEN DER KRANKENSCHWESTER IN BEZUG AUF DEN MOBILEN NOTFALLDIENST - SAMU
}

\section{INTEGRATIVE REVIEW}

REIS, Adilson dos ${ }^{1}$

EVARISTO, Leidiane da Silva ${ }^{2}$

SOUSA, Clarisse Cristina Ferreira Silva ${ }^{3}$

CASTRO, Mônica França de ${ }^{4}$

GUASTALDI, Rosimeire ${ }^{5}$

REIS, Adilson dos. Et al. Die Kompetenzen der Krankenschwester in Bezug auf den mobilen Notfalldienst - SAMU. Revista Científica Multidisciplinar Núcleo do Conhecimento. 04-Jahr, Ed. 11, Band 04, S. 159-170. November 2019. ISSN: 24480959,

Zugangslink: https://www.nucleodoconhecimento.com.br/gesundheit/kompetenzender-krankenschwester

\section{ZUSAMMENFASSUNG}

Einleitung: In Brasilien werden Notfall- und Notfallversorgungsnetze durch das einheitliche Gesundheitssystem unterstützt und reguliert, der Pflegefachmann ist für

\footnotetext{
${ }^{1}$ Abschluss in Krankenpflege.

2 Studium der Krankenpflege.

${ }^{3}$ Studium der Krankenpflege.

${ }^{4}$ Studium der Krankenpflege.

${ }^{5}$ Promotion in Gesundheitswissenschaften. Master in Gesundheitswissenschaften.

Spezialisierung im Pflegedienstmanagement. Bachelor-Pflege.
} 
die Pflegepraktiken mobiler Notfallversorgungsdienste (SAMU) ), nicht nur im Hinblick auf Beratung und medizinische Unterstützung, es wird darauf hingewiesen, dass die Pflege eine große Anzahl von Funktionen im Zusammenhang mit seiner Pflege in SAMU hat. Ziele: Ermittlung der Arbeit von Pflegekräften im Zusammenhang mit dem Mobilen Notfalldienst. Methode: Es wurde eine systematische Literaturrecherche durchgeführt, mit der Analyse der wichtigsten Pflegezeitschriften in den Scientific Electronic Library Online-Datenbanken (SciELO), Lateinamerikanischer und karibischer Literatur in Gesundheitswissenschaften (Lilacs) und dem Online-System für die Suche und Analyse medizinischer Literatur (Pubmed/MEDLINE). Insgesamt wurden 47 Artikel zum Thema der Studie gesammelt. Ergebnisse: Es wurde nachgewiesen, dass es eine beträchtliche Anzahl von Artikeln im Zusammenhang mit den Kompetenzen der Krankenschwester gegenüber SAMU und die Relevanz des Berufs für das ordnungsgemäße Funktionieren von SAMU gibt. Fazit: Es wird der Schluss gezogen, dass durch die wissenschaftliche und technische Entwicklung des Berufs, mit der Durchführung von Bachelor- und Graduiertenkursen, Pflegezuschreibungen in Bezug auf SAMU als wichtig angesehen werden, dass aber weitere Forschung von Studenten dieser Kategorie.

Schlagworte: Pflege, Mobiler Notfalldienst, Pflegeaufgaben.

\section{EINFÜHRUNG}

In Brasilien werden Notfall- und Notfallversorgungsnetze durch das einheitliche Gesundheitssystem, Verordnung n 1.600, 2011, unterstützt und reguliert. Und sie werden von den Segmenten Gesundheitsförderung, Prävention und Überwachung sowie mobilen Notfalldiensten (SAMU) etabliert. Krankenschwestern sind die Fachkraft mit wissenschaftlicher Ausbildung und menschlicher Kompetenz im Umgang mit dem Leben, und ihre Rolle ist von größter Bedeutung für die Pflege, die invasive Verfahren in Fällen mit fortgeschrittenen lebenserhaltenden Einheiten (VAS) erfordert. 
Die Krankenpflege ist eine breite Wissenschaft, die Menschenrechte und Gesundheit in Betracht zieht, mit einem humanisierten Blick auf die menschliche Entwicklung und erobert zunehmend ihren Raum in den unterschiedlichsten Gesundheitssektoren. Früher galt der Pflegefachmann als Hilfsfachmann des Arztes, heute wird dieser Beruf mit Amplitude verstanden, der mehrere Beiträge hat, die Patienten begünstigen, sei es über Information, Verfahren, Umgang mit Ausrüstung und Humanisierung. ${ }^{2}$

Der Pflegefachmann ist wesentlich für die von SAMU durchgeführten Pflegepraktiken, nicht nur in Bezug auf Beratung und medizinische Unterstützung, sondern auch in der humanisierten Pflege, mit einer breiten Vision von Organisation, Teammanagement, erfüllt Ausrüstung und Manöver, die Leben retten. Daher ist es möglich, dass sich Pflegekräfte ständig auf weiterbildung konzentrieren, so dass sie sich zunehmend auf die Erstversorgung spezialisiert, um ihre Arbeit mit Qualität und Effektivität zu entwickeln. ${ }^{3}$

Laut Costa 2016 sollte die Leistung der Krankenschwester in der mobilen vorstationären Versorgung ausgebaut werden, denn es ist ein extrem wichtiger Bereich für das Leben und je effizienter diese Versorgung ist, desto größer ist die Chance auf das Leben eines Menschen. Dementsprechend stellt der Mobile Emergency Care Service - SAMU für Mendes 2010 eine entscheidende Betreuung für den Einzelnen dar, der durch einige gesundheitliche Komplikationen geht und von Fachleuten begleitet werden muss, die fit sind und in der Lage sind, alle erforderlichen Verfahren zur Erhaltung des Lebens ${ }^{3}$

Die Qualität der in Brasilien in den 80er Jahren angebotenen Gesundheitsdienstleistungen hat sich erheblich verändert, was auf den Mangel an Finanzmitteln zurückzuführen ist, die die Bedeutung einer neuen Art der Verwaltung und Verwaltung der Finanzmittel begründen. Das Qualitätsmanagement der Pflege stellt eine große Bedeutung dar, daher wird es als eine Strategie verstanden, die einen akzeptablen kontinuierlichen Standard der Qualitätspflege erreicht. Management 
ermöglicht die Entwicklung von Humanressourcen, die Verantwortung für das Team, einschließlich Manager und Administratoren, schaffen ${ }^{4}$

Da Pflegekräfte in der vorstationären Versorgung unverzichtbar sind, wird es von großem Interesse, die Kompetenzen und Beiträge für den Bereich zu verstehen, stellt sich die Frage: Was sind die Zuschreibungen der Krankenschwester in Bezug auf die Pflege in der Samu?

\section{THEORETISCHEN RAHMEN}

Die hohe Nachfrage nach effektiven Notfalldiensten und medizinischen Notfällen hat weltweit zu mehreren Modellen von Pflegesystemen hervorgebracht. In Brasilien fand der erste Vorschlag 2003 über die Nationale Politik der Aufmerksamkeit für Notfälle (PNAU) statt $^{5}$

Laut Mendes 2010 stellt diese Politik die Bemühungen des Gesundheitsministeriums dar, regionale Ströme der umfassenden Versorgung von Dringend- und Notfällen in Brasilien zu organisieren. So orientiert sich die PNAU an den Prinzipien des SUS und ist in fünf Achsen gegliedert, die: Förderung der Lebensqualität; Vernetzung; Betrieb von Regulierungsanlagen; Aus- und Weiterbildung; Humanisierung der Aufmerksamkeit. ${ }^{6}$

Die Umsetzung war geprägt von der mobilen vorstationären Komponente, die sich als Strategie im Mobilen Notfalldienst (SAMU) präsentierte und ein standardisiertes Versorgungsmodell darstellt, in dem die Versorgung nach einem Anruf kostenlos für die Nummer 192, und das medizinische Zentrum legt fest, wie die Pflege durchgeführt werden soll. ${ }^{6}$

Darüber hinaus wurde der Zugang zu Dienstleistungen erweitert, nicht nur durch den Service der SAMU, sowie durch die Implementierung der Notfallversorgungseinheiten (UPA), die sich als die jüngste Strategie der Politik präsentieren und die Strategie der Aus- und Weiterbildung von Fachkräften, genannt Kerne und Bildung und Dringlichkeit. ${ }^{6}$ 


\section{ERGEBNISSE}

In der Suche nach der Bedeutung von Krankenschwestern in Bezug auf den mobilen Notdienst (SAMU) wurden bei der Datenerhebung unter Berücksichtigung der Inklusions- und Ausschlusskriterien 9 wissenschaftliche Artikel gefunden, die sich mit den Kompetenzen befassten. Krankenschwestern pflege und Management-Sekten in Veröffentlichungen in den Tabellen des vorherigen Artikels vorgestellt, in zwei Stufen, die erste sagt über die Charakterisierung der veröffentlichten Artikel über den Titel, Ort, Autor, Jahr, Art der Studie und Ziel und die zweite über die Fähigkeiten und Aktivitäten und prozentualen Anteil.

Die Datenerhebung dieser integrativen Überprüfung ergab die Auswahl der in Tabelle 1 beschriebenen Studien.

Tabelle 1 - Charakterisierung veröffentlichter Artikel über Titel, Ort, Autor, Jahr, Art der Studie und Ziel. São Paulo, 2019

\begin{tabular}{|c|c|c|c|c|}
\hline $\begin{array}{l}\text { Identifizier } \\
\text { ung }\end{array}$ & Titel, autor & Ort, Jahr & Art der Studie & Ziel \\
\hline A1 & $\begin{array}{l}\text { Krankenschwestern } \\
\text { des mobilen } \\
\text { Notfalldienstes: Profil } \\
\text { und Aktivitäten } \\
\text { entwickelt. } \\
\text { Luchtemberg MN, } \\
\text { Pires DEP }\end{array}$ & $\begin{array}{l}\text { Florianóp } \\
\text { olis, } 2015\end{array}$ & $\begin{array}{l}\text { Qualitative } \\
\text { beschreibend } \\
\text { e Studie }\end{array}$ & $\begin{array}{l}\text { Charakterisieren } \\
\text { Sie das Profil und } \\
\text { identifizieren Sie } \\
\text { die Aktivitäten, die } \\
\text { von } \\
\text { Krankenschweste } \\
\text { rn aus dem } \\
\text { mobilen } \\
\text { Notfalldienst eines } \\
\text { Staates } \\
\text { Südbrasilien in } \\
\text { entwickelt wurden }\end{array}$ \\
\hline
\end{tabular}




\begin{tabular}{|c|c|c|c|c|}
\hline A2 & $\begin{array}{l}\text { Weiterbildung als } \\
\text { Managementstrategie } \\
\mathrm{n} \text { im mobilen } \\
\text { Notfalldienst. Hetti } \\
\text { LBE, Bernardes A, } \\
\text { Gabriel CS, Fortuna } \\
\text { CM, Maziero VG. }\end{array}$ & $\begin{array}{l}\text { São } \\
\text { Paulo, } \\
2013\end{array}$ & $\begin{array}{l}\text { Qualitative } \\
\text { Studie }\end{array}$ & $\begin{array}{l}\text { Die Studie zielte } \\
\text { darauf ab, die } \\
\text { Wahrnehmung } \\
\text { von Fachleuten } \\
\text { über die ständige } \\
\text { und/oder } \\
\text { Weiterbildung im } \\
\text { Mobilen } \\
\text { Notfalldienst einer } \\
\text { Gemeinde im } \\
\text { Bundesstaat Sao } \\
\text { Paulo } \\
\text { analysieren. }\end{array}$ \\
\hline A3 & $\begin{array}{l}\text { Die Ansicht der } \\
\text { Krankenschwester/M } \\
\text { anagerin über die } \\
\text { Notwendigkeit, } \\
\text { psychologische } \\
\text { Unterstützung für } \\
\text { Fachkräfte im mobilen } \\
\text { Notfalldienst zu } \\
\text { implementieren; } \\
\text { Meskita KL, Gomes } \\
\text { GPLA, Silva MJBF, } \\
\text { Santos LF. }\end{array}$ & $\begin{array}{l}\text { Tocantins } \\
\text {, } 2014 .\end{array}$ & $\begin{array}{l}\text { Beschreibend } \\
\text { e und } \\
\text { explorative } \\
\text { qualitative } \\
\text { Forschung }\end{array}$ & $\begin{array}{l}\text { Analysieren Sie } \\
\text { die Sicht der } \\
\text { Krankenschweste } \\
\mathrm{r} / \mathrm{ge} \text { auf die } \\
\text { Notwendigkeit, } \\
\text { psychologische } \\
\text { Unterstützung für } \\
\text { SAMU-Profis zu } \\
\text { implementieren }\end{array}$ \\
\hline A4 & $\begin{array}{l}\text { Der Alltag von } \\
\text { Krankenschwestern, } \\
\text { die im mobilen } \\
\text { Notfalldienst arbeiten. } \\
\text { Tavares TY, Santana }\end{array}$ & $\begin{array}{l}\text { Minas } \\
\text { Gerais, } \\
2017\end{array}$ & $\begin{array}{l}\text { Qualitative } \\
\text { und } \\
\text { explorative } \\
\text { Forschung }\end{array}$ & $\begin{array}{l}\text { Verständnis der } \\
\text { täglichen Arbeit } \\
\text { von } \\
\text { Krankenschweste } \\
\text { rn, die im Mobilen }\end{array}$ \\
\hline
\end{tabular}




\begin{tabular}{|c|c|c|c|c|}
\hline & $\begin{array}{l}\text { JCB, Eloy MD, } \\
\text { Oliveira RD, Paula RF }\end{array}$ & & & $\begin{array}{l}\text { Notfalldienst } \\
\text { arbeiten }\end{array}$ \\
\hline A5 & $\begin{array}{l}\text { Perspektiven von } \\
\text { Pflegekräften in der } \\
\text { Pflege in der mobilen } \\
\text { vorstationären Pflege. } \\
\text { Anjos MS, Oliveira } \\
\text { SS, Rosa DOS }\end{array}$ & $\begin{array}{l}\text { Bahia, } \\
2016\end{array}$ & $\begin{array}{l}\text { Qualitative } \\
\text { Sondierungss } \\
\text { tudie }\end{array}$ & $\begin{array}{l}\text { Beschreiben Sie } \\
\text { die Perspektiven } \\
\text { von Pflegekräften } \\
\text { in der Pflege in der } \\
\text { mobilen } \\
\text { vorstationären } \\
\text { Pflege. }\end{array}$ \\
\hline A6 & $\begin{array}{l}\text { Leistungen von } \\
\text { Krankenschwestern in } \\
\text { der mobilen } \\
\text { vorstationären } \\
\text { Versorgung. Adão } \\
\text { RS, Santos MR. }\end{array}$ & $\begin{array}{l}\text { São } \\
\text { Paulo, } \\
2013\end{array}$ & $\begin{array}{l}\text { Qualitative } \\
\text { bibliographisc } \\
\text { he Bewertung }\end{array}$ & $\begin{array}{l}\text { Ziel dieser Studie } \\
\text { war es, die } \\
\text { Handlungen von } \\
\text { Krankenschweste } \\
\text { rn und } \\
\text { Krankenschweste } \\
\text { rn in einer } \\
\text { grundlegenden } \\
\text { und } \\
\text { fortgeschrittenen } \\
\text { Gesundheitseinhe } \\
\text { it in mobiler PHA } \\
\text { durch literarische } \\
\text { Überprüfung zu } \\
\text { beschreiben. }\end{array}$ \\
\hline A7 & $\begin{array}{l}\text { Die Leistung der } \\
\text { Krankenschwester } \\
\text { eines privaten } \\
\text { vorstationären } \\
\text { Pflegedienstes. Peres } \\
\text { PSQ, Arboit EL, } \\
\text { Camponogara S, }\end{array}$ & $\begin{array}{l}\text { Rio } \\
\text { Grande } \\
\text { do Sul, } \\
2018\end{array}$ & $\begin{array}{l}\text { Qualitative } \\
\text { explorative } \\
\text { beschreibend } \\
\text { e Studie }\end{array}$ & $\begin{array}{l}\text { Die } \\
\text { Wahrnehmung } \\
\text { von } \\
\text { Gesundheitsperso } \\
\text { nal über die } \\
\text { Leistung der } \\
\text { Krankenschweste }\end{array}$ \\
\hline
\end{tabular}




\begin{tabular}{|c|c|c|c|c|}
\hline & $\begin{array}{l}\text { Pilau COB, Menezes } \\
\text { OS, Kaefer CT. }\end{array}$ & & & $\begin{array}{l}r \quad \text { in einem } \\
\text { vorstationären } \\
\text { Pflegedienst zu } \\
\text { kennen. }\end{array}$ \\
\hline A8 & $\begin{array}{l}\text { Leistungen von } \\
\text { Krankenschwestern in } \\
\text { der mobilen } \\
\text { vorstationären } \\
\text { Versorgung. } \\
\text { Fernandez MG, } \\
\text { Espindula BM. }\end{array}$ & $\begin{array}{l}\text { São } \\
\text { Paulo, } \\
2018\end{array}$ & $\begin{array}{l}\text { Integrative } \\
\text { Literaturscha } \\
\text { u }\end{array}$ & $\begin{array}{l}\text { Ziel dieser Studie } \\
\text { war es, die } \\
\text { Handlungen von } \\
\text { Krankenschweste } \\
\text { rn ind einer } \\
\text { Krankenschweste } \\
\text { rn in } \\
\text { grundlegenden } \\
\text { und } \\
\text { fortgeschrittenen } \\
\text { Gesundheitseinhe } \\
\text { it in mobiler PHA } \\
\text { durch literarische } \\
\text { Überprüfung zu } \\
\text { beschreiben. }\end{array}$ \\
\hline A9 & $\begin{array}{l}\text { Betreuung von } \\
\text { Pflegekräften in der } \\
\text { mobilen } \\
\text { vorstationären } \\
\text { Versorgung. } \\
\text { Bernardes A, Maziero } \\
\text { GV, Hetti LBE, Baldin } \\
\text { MSC, Gabriel CS. }\end{array}$ & $\begin{array}{l}\text { São } \\
\text { Paulo, } \\
2014\end{array}$ & $\begin{array}{l}\text { Qualitative } \\
\text { beschreibend } \\
\text { e Forschung }\end{array}$ & $\begin{array}{l}\text { Die Studie zielte } \\
\text { darauf ab, zu } \\
\text { analysieren, wie } \\
\text { die Überwachung } \\
\text { im Mobilen } \\
\text { Notfallversorgung } \\
\text { sdienst (SAMU) } \\
\text { einer Gemeinde } \\
\text { im Bundesstaat } \\
\text { Sao Paulo } \\
\text { stattfindet. }\end{array}$ \\
\hline
\end{tabular}


Quelle: Kings, 2019.

Die ausgewählten Studien wurden analysiert und nach den in Schaubild 2 beschriebenen Kompetenzen, Aktivitäten und Prozentgruppiert.

Tabelle 2: Verteilung der gefundenen Artikel über die Betreuungs- und Managementkompetenzen. São Paulo, 2019

\begin{tabular}{|c|c|c|c|}
\hline Тур & Artikel & Aktivitäten & $\%$ \\
\hline Hilfe & $\begin{array}{l}\text { A5 } \\
\text { A6 } \\
\text { A7 } \\
\text { A8 }\end{array}$ & $\begin{array}{l}\text { - } \text { Beurteilung von Verletzungen und Behandlung; } \\
\text { - } \quad \text { Medikamente als Rezept; } \\
\text { - Venöse Zugangspunktion, Blase und } \\
\text { nasogastrische } \\
\text { - Herzüberwachung und Pulsoximetrie; } \\
\text { - Hämodynamische Auswertung, mechanische } \\
\text { Belüftungspflege; } \\
\text { - Dressings; }\end{array}$ & $66,6 \%$ \\
\hline Management & $\begin{array}{l}\text { A2 } \\
\text { A3 } \\
\text { A9 }\end{array}$ & $\begin{array}{l}\text { - Managementtätigkeiten } \\
\text { Verwaltungstätigkeiten; } \\
\text { - Durchführung von Schulungen und dauernder } \\
\text { Ausbildung; } \\
\text { - Erstellen von } \\
\text { - Überwachung und Bewertung der Aktivitäten des } \\
\text { Teams in mobilem APH; } \\
\text { - Vervollständigung des Fallblattes; } \\
\text { - Checkliste der Materialien, Arzneimittel und } \\
\text { Ausrüstung; } \\
\text { - Bericht in einem Buchprotokoll, } \\
\text { Bestandsaufnahme der Basis- } \\
\text { Bestandskontrolle, Pflegebericht, Anfrage nach } \\
\text { Arzneimitteln und } \\
\text { - Desinfektion von Ausrüstung und Fahrzeug; }\end{array}$ & $33,3 \%$ \\
\hline
\end{tabular}




\begin{tabular}{|c|c|c|}
\hline & $\begin{array}{l}\text { - Kontrollierte } \\
\text { Übertragungsverantwortung Begriff, Lager } \\
\text { Temperaturkontrolle; } \\
\text { - Umfang der Desinfektion des Fahrzeugs, } \\
\text { Ausfüllen von Krankenakten, Teilnahme an der } \\
\text { Ausarbeitung von Projekten wie der samu- } \\
\text { Ausbildung; } \\
\text { - Teilnahme an Sitzungen und Ausfüllen des } \\
\text { Punkteblattes }\end{array}$ & \\
\hline gesamt: & & $100 \%$ \\
\hline
\end{tabular}

Quelle: Könige, 2019

\section{DISKUSSION}

Um die Ergebnisse besser zu verstehen, werden sie in zwei Themen erörtert: Pflegefähigkeiten für die Leistung der Krankenschwester in SAMU und Managementfähigkeiten für die Rolle der Krankenschwester in SAMU.

\section{CARE SKILLS VON NURSES ZU ACT IN SAMU}

Die Veröffentlichungen befassten sich mit den Kompetenzen der Krankenschwester, sowohl Pflege als auch Management gleichzeitig, aber 33\% $(\mathrm{n}=3)$ der Publikationen, der Schwerpunkt lag auf der Leistung der Krankenschwester als Managerin, unter den Pflegefähigkeiten gefunden, die am meisten abheben und sind wiederkehrende sind: Bewertung des Ausmaßes der Krankheiten, die Priorität für ein größeres Engagement und die Leistung der Verfahren und Pflege. Ausstehend unter den Verfahren, venöse Punktion, Durchgang der Blase Schneidrohr und nasogastric Sondierung, während in der Bereitstellung von Pflege gefunden wurde: Leistung der Verbände, Überwachung der Vitalzeichen und Verabreichung von Medikamenten, Herzüberwachung und Pulsoximetrie, Hygiene und Komfort, hämodynamische Auswertung, 
Immobilisierungen, Anamnese, ärztliche Assistenz bei Eingriffen wie Reanimation und Entscheidungsfindung. ${ }^{6}$

Unter anderem. Angesichts der vielfältigen Funktionen, die von der Krankenschwester ausgeführt werden, ist es klar, die Idee der Bedeutung der Krankenschwester und die Verantwortung, die inm übertragen wird, die die Entwicklung von Fähigkeiten erfordert, die Kognition, Haltungen und die Durchführung der Verfahren, die durch endet durch in der Rolle des Managers führen, was in vielen Fällen das Entstehen körperlicher und geistiger Erschöpfung bedeutet. Daher ist es wichtig, menschliche und physische Ressourcen zur Verfügung zu stellen, um das Opfer bestzuseint zu versorgen, immer mit dem Ziel, die Probleme zu minimieren.

\section{MANAGERIAL SKILLS VON NURSES IN SAMU ARBEITEN}

Für die wichtigsten Management-Kompetenzen wurde nachgewiesen, dass es die Aufgabe der Krankenschwestern ist, Protokolle für die korrekte Versorgung zu entwickeln, unter Berücksichtigung aller Stichproben, die die Pflege und das Engagement des Patienten rechtfertigt.8 Auch mit der des Patienten. Krankenschwester, das Ausfüllen des Anwesenheitsformulars, die Überprüfung und Auffüllung des Materials, das sich im Fahrzeug befinden muss und für die Pflege, Desinfektion von Ausrüstung und Fahrzeug, Pflegebericht, Teilnahme oder Entwicklung von Projekten, die Gesundheitserziehung dauerhaft ermöglichen ${ }^{9}$

Angesichts der Nachgewiesenkeit der Kompetenzen der Krankenschwestern wird die Bedeutung und die Tatsache verstanden, dass dieser Fachmann sowohl unter Berücksichtigung der Pflege- und Führungstätigkeiten, die der Krankenschwester beraubt werden, als auch der Unterstützung der Mitarbeiter der Krankenschwestern und Pflegeteam, das zum Qualitätsindex der Pflegeleistung führt, versteht, dass NichtLeistung, Nicht-Wissen ihrer relevanten Aktivitäten Schäden oder Tod für das opfer besuchte Charakterisierung charakterisieren kann. ${ }^{9}$

Über alles hinaus zielt die gute Qualität der Pflege auf die Pflege, die das Pflegeteam leitet, und das gleiche sollte für die Größe des Teams verantwortlich sein, unter 
Berücksichtigung der Leistung jedes Mitarbeiters, der Ausarbeitung Protokolle, die auf wissenschaftlichen Erkenntnissen basieren und an die Realität der brasilianischen Gesundheit angepasst sind, Wege zur Durchführung von Pflege mitteln und die die Handlungen des Profis unterstützen.10 Laut Santos und Adam, 2013 die Einführung der professionellen Krankenschwester in der Pflege Mobile pre-hospital wurde durch die PNAU gegeben, die sich mit dem, was der Regionale Pflegerat sagt, einig ist, die diese professionelle wichtig für die Zusammensetzung des PHA-Teams, da es in der Lage ist, Maßnahmen mit dem Ziel der Schäden zu erhalten und zu verringern, entweder durch direkte Hilfe oder Teammanagement. ${ }^{10}$

Als Manager ist eine der wichtigsten Aufgaben der Krankenschwestern die Weiterbildung im Gesundheitswesen, da sie über theoretische und wissenschaftliche Kenntnisse verfügt. Diese Gesundheitserziehung wird durch die Anleitungunden und Erklärungen, die an die Familie des Opfers weitergegeben werden, eher routinemäßig übersetzt als an die Pflegekräfte, bei denen Krankenschwestern für die Aus- und Weiterbildung ihrer Mitarbeiter zuständig sind, um bessere Qualität der erbrachten Dienstleistungen ${ }^{1,10}$

\section{FAZIT}

Von den 9 Artikeln, die zur Erreichung der vorgeschlagenen Ziele ermittelt wurden, befassten sich $100 \%(n=9)$ mit Pflegekompetenzen, aber von diesen insgesamt befassten sich nur 33\% ( $n=3)$ mit den Managementkompetenzen der Pflegekategorie. Bei den Managementkompetenzen fielen diejenigen auf, die eine direkte Beziehung zum Opfer haben, wie die Bereitstellung von Pflegeleistungen. Während Manager Fähigkeiten fanden, die nicht direkt mit dem Opfer verbunden sind, mit administrativen Aktivitäten als die wichtigsten, wie Team-Größe. Es wird der Schluss gezogen, dass Krankenschwestern ein unverzichtbarer Fachmann sind, sowohl in der direkten Betreuung des Opfers als auch in den Aktionen, die diese Pflege koordinieren. 


\section{VERWEISE}

1. LUCHTEMBERG, Marilene Nonnemacher; PIRES, Denise Elvira Pires de. Enfermeros del Servicio de Atencién Mévil de Urgencia: profile y desarrolladas activities. Brazilian Journal of Nursing, v. 69, n. 2, S. 213-220, 2016.

2. Adam, Rodrigo de Souza; SANTOS, Maria Regina dos. Leistungen von Krankenschwestern in der mobilen vorstationären Versorgung. Revista Mineira de Enfermagem, v. 16, n. 4, S. 601-608, 2012.

3. BERNARDES, Andrea et al. Betreuung von Pflegekräften in der mobilen vorstationären Versorgung. Electronic Nursing Journal, v. 16, n. 3, S. 635-43, 2014.

4. BRASILIEN, Gesundheitsministerium. Nationale Politik für die Notfallversorgung. Brasilia, Redakteurin des Gesundheitsministeriums, 3. ed. ampl., 2006.

5. COSTA, Gabriela. Ein Ansatz für die Pflegegeschichte Maßnahmen im Angesicht der Gesundheitspolitik. Revista Mineira de Enfermagem - REME; Band 10.4; Joo Pessoa - PB, 2016

6. EL HETTI, Livia Barrionuevo et al. Weiterbildung als Managementstrategien im Mobilen Notfalldienst. Electronic Nursing Journal, v. 15, n. 4, S. 973-82, 2013.

7. MENDES, Eugénio Vilaa. Gesundheitsnetze. Ciénz. kollektive Gesundheit, Rio de Janeiro, v. 15, n. 5, S. 2297-2305, Aug. 2010.

8. MESQUITA, Kayena Lopes et al. Die Sorgekraft/Leiterin sieht die Notwendigkeit, professionelle Fachkräfte im mobilen Notfalldienst psychologisch zu unterstützen. Krankenjournal des Mittleren Westens von Minas Gerais, 2014.

9. NETTINA, Sandra ( Pflegepraxis. 9. Ed. Rio de Janeiro: Guanabara Koogan, 2011.

10. PERES, Paulo Sergio Quevedo et al. Leistungen von Krankenschwestern in einem privaten vorstationären Pflegedienst/Krankenschwesternleistung 
auf einer privaten vorstationären Betreuung. Research Magazine: Caution is Fundamental Online, v. 10, n. 2, S. 413-422, 2018.

11. TAVARES, Tayrine Ypuena et al. Der Alltag von Krankenschwestern, die im mobilen Notfalldienst arbeiten. Nursing Journal of The Midwest of Minas Gerais, v. 7, 2017.

Eingereicht: November 2019.

Genehmigt: November 2019. 